PROCEEDINGS OF THE

AMERICAN MATHEMATICAL SOCIETY

Volume 135, Number 1, January 2007, Pages 181-189

S 0002-9939(06)08467-X

Article electronically published on June 28, 2006

\title{
POINTWISE CONVERGENCE OF BOUNDED CASCADE SEQUENCES
}

\author{
DI-RONG CHEN AND MIN HAN \\ (Communicated by David R. Larson)
}

\begin{abstract}
The cascade algorithm plays an important role in computer graphics and wavelet analysis. For an initial function $\phi_{0}$, a cascade sequence $\left(\phi_{n}\right)_{n=0}^{\infty}$ is constructed by the iteration $\phi_{n}=C_{a} \phi_{n-1}, n=1,2, \ldots$, where $C_{a}$ is defined by $C_{a} g=\sum_{\alpha \in \mathbb{Z}} a(\alpha) g(2 \cdot-\alpha), g \in L_{p}(\mathbb{R})$. In this paper, under a condition that the sequence $\left(\phi_{n}\right)_{n=0}^{\infty}$ is bounded in $L_{\infty}(\mathbb{R})$, we prove that the following three statements are equivalent: (i) $\left(\phi_{n}\right)_{n=0}^{\infty}$ converges a.e. $x \in \mathbb{R}$. (ii) For a.e. $x \in \mathbb{R}$, there exist a positive constant $c$ and a constant $\gamma \in(0,1)$ such that $\left|\phi_{n+1}(x)-\phi_{n}(x)\right| \leq c \gamma^{n} \forall n=1,2, \ldots$ (iii) For some $p \in[1, \infty),\left(\phi_{n}\right)_{n=0}^{\infty}$ converges in $L_{p}(\mathbb{R})$. An example is presented to illustrate our result.
\end{abstract}

\section{INTRODUCTION}

We are interested in the functional equation of form

$$
\phi=\sum_{\alpha \in \mathbb{Z}} a(\alpha) \phi(2 \cdot-\alpha),
$$

where $a=(a(\alpha))_{\alpha \in \mathbb{Z}}$, called a refinement mask, is a finitely supported sequence on $\mathbb{Z}$. The equation is known as a refinement equation. A nonzero function $\phi$ is a refinable function corresponding to $a$ if it satisfies the above equation.

Associated with the above refinement equation is the cascade operator $C_{a}$ defined by

$$
C_{a} g=\sum_{\alpha \in \mathbb{Z}} a(\alpha) g(2 \cdot-\alpha), \quad g \in L_{p}(\mathbb{R}) .
$$

For any initial function $\phi_{0}$, we construct a sequence by the iteration

$$
\phi_{n}=C_{a} \phi_{n-1}=C_{a}^{n} \phi_{0}, \quad n=1,2, \ldots
$$

This sequence is called a cascade sequence generated by $\phi_{0}$. Such a construction method of sequences is known as the cascade algorithm. It is the most powerful tool to solve the refinement equations.

The cascade algorithm plays an important role in computer graphics and wavelet analysis. The convergence of the subdivision scheme and cascade algorithm in

Received by the editors February 10, 2005 and, in revised form, July 29, 2005.

2000 Mathematics Subject Classification. Primary $42 \mathrm{C} 15$.

Key words and phrases. Refinable function, cascade algorithm, subdivision scheme, pointwise convergence, refinable curve, joint spectral radius.

This research was supported in part by NSF of China under grant 10571010 . 
$W_{p}(\mathbb{R})$ and the Sobolev space $W_{p}^{s}(\mathbb{R})$ has been studied by many authors. We mention here some work, but without any completeness at all. Cavaretta et al. 2] already found necessary and sufficient conditions ensuring that the cascade algorithm converges uniformly to a continuous limit function. In $L_{2}$ the convergence had been shown by Strang [21, Lawton et al. [14, 20] and [16. Jia 9] considered the convergence of cascade algorithms in the univariate setting for general $L_{p^{-}}$-spaces. For the univariate vector case we refer to Jia et al. 12 and to Micchelli et al. 18. Chen et al. [6] and Li [15, studied this problem in the general setting. The convergence of cascade algorithms for perturbed refinement masks was discussed in 7. Recently, with the help of the cascade algorithm, an easily implemented algorithm for the construction of smooth refinable functions in $L_{p}(\mathbb{R}), 1 \leq p<\infty$, was presented in 3 .

However, as far as we know, there has been very little discussion on $L_{\infty}$ refinable functions or the pointwise convergence of cascade sequences in $L_{\infty}(\mathbb{R})$ in literature. The properties of $L_{\infty}$ refinable functions are somewhat different from those of refinable functions in $W_{p}(\mathbb{R})$. For example, if a compactly supported refinable function $\phi \in W_{p}(\mathbb{R})$, then

$$
\|\phi-\phi(\cdot-h)\|_{W_{p}(\mathbb{R})}=O\left(h^{s}\right), \quad \text { as } h \rightarrow 0,
$$

where $s>0$ is a constant. It is an interesting question of whether the analogous conclusion holds for the $L_{\infty}$ case. The author established a result about pointwise regularity of an arbitrary $L_{\infty}$ refinable function in 4 . This result may be interpreted as a partially positive answer to the above question in the pointwise case.

In this paper we study the pointwise convergence of a bounded cascade sequence in $L_{\infty}(\mathbb{R})$. Our main result is the following.

Theorem 1. Let $\phi_{0}$ be a compactly supported function in $L_{\infty}(\mathbb{R})$. Suppose that the cascade sequence $\left(\phi_{n}\right)_{n=0}^{\infty}$ is bounded in $L_{\infty}(\mathbb{R})$. Then the following statements are equivalent:

(i) $\left(\phi_{n}\right)_{n=0}^{\infty}$ converges a.e. $x \in \mathbb{R}$.

(ii) For a.e. $x \in \mathbb{R}$, there exist a positive constant $c$ and a constant $\gamma \in(0,1)$ such that $\left|\phi_{n+1}(x)-\phi_{n}(x)\right| \leq c \gamma^{n} \forall n=1,2, \ldots$

(iii) For some $p \in[1, \infty),\left(\phi_{n}\right)_{n=0}^{\infty}$ converges in $L_{p}(\mathbb{R})$.

The paper is organized as follows. In Section 2 we consider the pointwise convergence in a setting more general than the cascade algorithm. As a corollary of the results of Section 2, the proof of Theorem 1 is given in Section 3. We also generalize Theorem 1 to the sequence of derivatives. Finally, an example is presented to illustrate our results.

\section{Convergence in the Setting of Refinable CURves}

We define a shift operator $\tau$ on $[0,1]$ by

$$
\tau x= \begin{cases}2 x, & 0 \leq x \leq 1 / 2, \\ 2 x-1, & 1 / 2<x \leq 1 .\end{cases}
$$

Let $x \in[0,1]$. There are $d_{j} \in\{0,1\}, j=1,2, \ldots$, such that $x=\sum_{j=1}^{\infty} 2^{-j} d_{j}$. This is a dyadic expansion of $x$, and $d_{j}$ is its $j$-th digit. The shift operator may be 
represented in terms of dyadic expansions as follows:

$$
\tau x=\sum_{j=1}^{\infty} 2^{-j} d_{j+1} \quad \text { for } x=\sum_{j=1}^{\infty} 2^{-j} d_{j} .
$$

If $x \notin\left\{t \in(0,1): t=k / 2^{m}, 0<k<2^{m}, m=1,2, \ldots\right\}$, the dyadic expansion of $x$ is unique. Consequently, for almost every $x \in[0,1]$, we may use $d_{j}(x)$ to denote its $j$-th digit.

To study refinable functions, it is sometimes convenient to work in a more general setting, the setting of refinable curves. Suppose that $A_{0}$ and $A_{1}$ are $N \times N$ matrices. A vector $\Phi$ of $N$ functions on [0,1] is referred to as a refinable curve (see e.g. [17]) if it satisfies

$$
\Phi(x)=A_{d_{1}(x)} \Phi(\tau x), \quad \text { a.e. on } x \in[0,1] .
$$

For an initial vector $\Phi_{0}$ of $N$ functions defined on $[0,1]$, the cascade sequence $\left(\Phi_{n}\right)_{n=0}^{\infty}$ generated by $\Phi_{0}$ is given by the iteration

$$
\Phi_{n}(x)=A_{d_{1}(x)} \Phi_{n-1}(\tau x), \quad \text { a.e. on } x \in[0,1] .
$$

Consequently,

$$
\Phi_{n}(x)=A_{d_{1}(x)} \cdots A_{d_{n}(x)} \Phi_{0}\left(\tau^{n} x\right), \quad \text { a.e. on } x \in[0,1] .
$$

Let $\|\cdot\|$ be a norm of $\mathbb{C}^{N}$. It derives a norm of the space of vectors $F$ of functions in $L_{p}([0,1])$ as follows:

$$
\|F\|_{p}=\left(\int_{0}^{1}\|F(x)\|^{p} d x\right)^{1 / p} .
$$

We say that the sequence $\left(\Phi_{n}\right)_{n=0}^{\infty}$ converges in $L_{p}$ if it converges in the norm $\|\cdot\|_{p}$. Clearly the definition is independent of the choice of the norm on $\mathbb{C}^{N}$.

Suppose that $A_{0}$ and $A_{1}$ are $N \times N$ matrices. A linear subspace $V \subseteq \mathbb{C}^{N}$ is called an $\left\{A_{0}, A_{1}\right\}$-invariant subspace if it is invariant under each $A_{d}, d=0,1$. For a subset set $S \subseteq \mathbb{C}^{N}$, denote by $V(S)$ the smallest $\left\{A_{0}, A_{1}\right\}$-invariant subspace containing $S$.

In our study, a special kind of set $S=S_{E, \Psi}$ given by

$$
S_{E, \Psi}=\{\Psi(x): x \in E\}
$$

will be used, where $E \subseteq[0,1]$ and $\Psi$ is a vector of $N$ functions on $[0,1]$.

Afterwards we use the same notation $\|\cdot\|$ to denote a norm of $\mathbb{C}^{N}$ and a matrix norm. The exact meaning should be clear from the context.

Lemma 2. Choose $\Phi_{0} \in\left(L_{\infty}([0,1])\right)^{N}$. Then the cascade sequence $\left(\Phi_{n}\right)_{n=0}^{\infty}$ is bounded in $\left(L_{\infty}([0,1])\right)^{N}$ if and only if there are a subset $E \subseteq[0,1]$ with $\mu(E)=1$ and a matrix norm $\|\cdot\|$ satisfying $\left\|\left.A_{d}\right|_{V\left(S_{E, \Phi_{0}}\right)}\right\| \leq 1, d=0,1$, where $S_{E, \Phi_{0}}$ is given as in (7).

Proof. We first verify the necessity. By assumption we have a subset $D \subseteq[0,1]$ with $\mu(D)=1$ and a constant $c$ such that $\left\|\Phi_{n}(x)\right\| \leq c \quad \forall x \in D$ and $n=1,2, \ldots$ Let $E=\bigcap_{n=1}^{\infty} \tau^{n}(D)$. Then $\mu(E)=1$. Moreover, we have by (6)

$$
\left\|A_{d_{1}} \cdots A_{d_{n}} \Phi_{0}(t)\right\| \leq c \quad \forall t \in E, d_{1}, \ldots, d_{n} \in\{0,1\} \text { and } n=1,2, \ldots
$$

Let $S=S_{E, \Phi_{0}}$ be defined by (7) and $\Sigma=\left\{\left.A_{d}\right|_{V(S)}: d=0,1\right\}$. By the last inequality, $\Sigma$ is product bounded. In other words, all products generated by $\Sigma$ are bounded uniformly. The necessity follows from [1]. 
Conversely, suppose that there are a subset $E \subseteq[0,1]$ with $\mu(E)=1$ and a matrix norm $\|\cdot\|$ satisfying $\left\|\left.A_{d}\right|_{V\left(S_{E, \Phi_{0}}\right)}\right\| \leq 1, d=0,1$. Let $D=\bigcap_{n=1}^{\infty} \tau^{-n}(E)$. Then $\mu(D)=1$ and for any $x \in D, \tau^{n} x \in E$.

Let $n_{0}=\operatorname{dim} V\left(S_{E, \Phi_{0}}\right)$. It is clear that $A_{d_{1}} \cdots A_{d_{n_{0}}} \Phi_{0}(t) \in V\left(S_{E, \Phi_{0}}\right)$ for any $t \in E$. Consequently, for $n \geq n_{0}$ and $x \in D$, we have, by (6) and the assumption on $A_{d}, d=0,1$, that $\left\|\Phi_{n}(x)\right\| \leq\left\|\Phi_{n_{0}}\left(\tau^{n-n_{0}} x\right)\right\| \leq\left\|\Phi_{n_{0}}\right\|_{\left(L_{\infty}[0,1]\right)^{N}}$. Therefore, the sequence $\left(\Phi_{n}\right)$ is bounded in $\left(L_{\infty}[0,1]\right)^{N}$. The proof is complete.

Before proceeding further, let us introduce the notion of joint spectral radius for the convenience of readers. Suppose that $V$ is a finitely dimensional vector space equipped with a vector norm $\|\cdot\|$. For a linear operator $A$ on $V$, define

$$
\|A\|:=\max \{\|A v\|:\|v\|=1\} \text {. }
$$

Let $\mathcal{A}$ be a finite set of linear operators on $V$. For a positive integer $n$ we denote by $\mathcal{A}^{n}$ the $n$-th Cartesian power of $\mathcal{A}$ :

$$
\mathcal{A}^{n}=\left\{\left(A_{1}, \ldots, A_{n}\right): A_{1}, \ldots, A_{n} \in \mathcal{A}\right\} .
$$

For $1 \leq p<\infty$, let

$$
\left\|\mathcal{A}^{n}\right\|_{p}:=\left(\sum_{\left(A_{1}, \ldots, A_{n}\right) \in \mathcal{A}^{n}}\left\|A_{1} \cdots A_{n}\right\|^{p}\right)^{1 / p},
$$

and for $p=\infty$ define

$$
\left\|\mathcal{A}^{n}\right\|_{\infty}:=\max \left\{\left\|A_{1} \cdots A_{n}\right\|:\left(A_{1}, \ldots, A_{n}\right) \in \mathcal{A}^{n}\right\} .
$$

For $1 \leq p \leq \infty$, the $p$-norm joint spectral radius of $\mathcal{A}$ is defined to be

$$
\rho_{p}(\mathcal{A}):=\lim _{n \rightarrow \infty}\left\|\mathcal{A}^{n}\right\|_{p}^{1 / n}
$$

It is easily seen that this limit indeed exists and

$$
\rho_{p}(\mathcal{A})=\inf _{n \geq 1}\left\|\mathcal{A}^{n}\right\|_{p}^{1 / n} .
$$

Clearly, $\rho_{p}(\mathcal{A})$ is independent of the choice of the vector norm on $V$.

The uniform joint spectral radius was introduced in [19]. The mean joint spectral radius was introduced in [22]. For $1<p<\infty$, the $p$-joint spectral radius was introduced in [9] and applied to the study of $L_{p}$ convergence of cascade algorithms.

Theorem 3. Let $\Phi_{0} \in\left(L_{1}([0,1])\right)^{N}$. Suppose that the sequence $\left(\Phi_{n}\right)_{n=0}^{\infty}$ converges in $\left(L_{1}([0,1])\right)^{N}$. Then for any matrix norm $\|\cdot\|$, there are a set $F \subseteq[0,1]$ with $\mu(F)=1$ and a positive integer $k$ such that

$$
\sum_{d_{1}, \ldots, d_{k}}\left\|B_{d_{1}} \ldots B_{d_{k}}\right\|<2^{k}
$$

where $B_{d}$ is the restriction of $A_{d}$ on $V(S)$, i.e., $B_{d}=\left.A_{d}\right|_{V(S)}, d=0,1$, and $S=$ $S_{F, \Phi_{1}-\Phi_{0}}$ is given as in (7).

Proof. For any $n$ and $d_{j} \in\{0,1\}, 1 \leq j \leq n$, define a subset $T_{d_{1} \ldots d_{n}}$ by

$$
T_{d_{1} \cdots d_{n}}=\left\{x: \sum_{j=1}^{n} 2^{-j-n} d_{j} \leq x \leq 2^{-n}+\sum_{j=1}^{n} 2^{-j-n} d_{j}\right\} .
$$

It follows from $\tau^{n}\left(T_{d_{1}, \ldots, d_{n}}\right)=[0,1]$ and (66) that

$$
\Phi_{n}(x)=A_{d_{1}} \cdots A_{d_{n}} \Phi_{0}\left(\tau^{n} x\right), \quad x \in T_{d_{1}, \ldots, d_{n}} .
$$


Consequently, for $x \in T_{d_{1} \cdots d_{n} d_{n+1}} \subset T_{d_{1} \cdots d_{n}}$,

$$
\begin{aligned}
\Phi_{n+1}(x)-\Phi_{n}(x) & =A_{d_{1}} \cdots A_{d_{n}}\left(A_{n+1} \Phi_{0}\left(\tau^{n+1} x\right)-\Phi_{0}\left(\tau^{n} x\right)\right) \\
& =A_{d_{1}} \cdots A_{d_{n}}\left(\Phi_{1}\left(\tau^{n} x\right)-\Phi_{0}\left(\tau^{n} x\right)\right),
\end{aligned}
$$

where the second equality is true due to $d_{1}\left(\tau^{n} x\right)=d_{n+1}(x) \forall x \in T_{d_{1} \cdots d_{n} d_{n+1}}$. Integrating on this subset gives

$$
\int_{T_{d_{1} \cdots d_{n} d_{n+1}}}\left\|\Phi_{n+1}(x)-\Phi_{n}(x)\right\| d x=2^{-n} \int_{\frac{d_{n+1}}{2}}^{\frac{d_{n+1}+1}{2}}\left\|A_{d_{1}} \cdots A_{d_{n}}\left(\Phi_{1}(x)-\Phi_{0}(x)\right)\right\| d x .
$$

Summing all the equalities over $d_{1}, \ldots, d_{n}, d_{n+1} \in\{0,1\}$ we have

$$
\left\|\Phi_{n+1}-\Phi_{n}\right\|_{1}=\int_{0}^{1} 2^{-n} \sum_{d_{1}, \ldots, d_{n}}\left\|A_{d_{1}} \cdots A_{d_{n}}\left(\Phi_{1}(x)-\Phi_{0}(x)\right)\right\| d x .
$$

The convergence of $\left(\Phi_{n}\right)_{n=0}^{\infty}$ in $\left(L_{1}([0,1])\right)^{N}$ and Fatou's Lemma imply

$$
\liminf _{n \rightarrow \infty} 2^{-n} \sum_{d_{1}, \ldots, d_{n}}\left\|A_{d_{1}} \cdots A_{d_{n}}\left(\Phi_{1}(x)-\Phi_{0}(x)\right)\right\|=0 \quad \text { a.e. }
$$

Let $F$ be the set of all the points $x \in[0,1]$ such that the above equality holds for $x$. Clearly, $\mu(F)=1$. For any $x \in F$, by the last equality and the method of 8], Lemma 2.4, we can conclude that $\rho_{1}\left(\left\{\left.A_{0}\right|_{V\left(S_{\{x\}, \Phi_{1}-\Phi_{0}}\right)},\left.A_{1}\right|_{V\left(S_{\{x\}, \Phi_{1}-\Phi_{0}}\right)}\right\}\right)<2$. Moreover, it is known from [5], Theorem 1, that

$$
\begin{aligned}
& \rho_{1}\left(\left\{\left.A_{0}\right|_{V\left(S_{F, \Phi_{1}-\Phi_{0}}\right)},\left.A_{1}\right|_{V\left(S_{F, \Phi_{1}-\Phi_{0}}\right)}\right\}\right) \\
& \quad=\max _{x \in F} \rho_{1}\left(\left\{\left.A_{0}\right|_{V\left(S_{\{x\}, \Phi_{1}-\Phi_{0}}\right)},\left.A_{1}\right|_{V\left(S_{\{x\}, \Phi_{1}-\Phi_{0}}\right)}\right\}\right) .
\end{aligned}
$$

Therefore, $\rho_{1}\left(\left\{B_{0}, B_{1}\right\}\right)<2$. By the definition of joint spectral radius, we obtain the existence of some $k$ such that (9) holds. The proof is complete.

For a fixed $k$-tuple $\left(d_{1}^{0}, \ldots, d_{k}^{0}\right) \in\{0,1\}^{k}$, a positive integer $n$ and $x \in[0,1]$, how many $i$ 's are there with $0 \leq i \leq[n / k]-1$ such that

$$
\left(d_{i k+1}(x), d_{i k+2}(x), \ldots, d_{i k+k}(x)\right)=\left(d_{1}^{0}, \ldots, d_{k}^{0}\right),
$$

where $[t]$ for $t \in \mathbb{R}$ denotes the largest integer smaller than or equal to $t$ ? The number of such $i$ 's is just the cardinality of the set

$$
\left\{0 \leq i \leq[n / k]-1: \tau^{i k} x \in T_{d_{1}^{0} \ldots d_{k}^{0}}\right\},
$$

since it is easily seen that (11) is equivalent to $\tau^{i k} x \in T_{d_{1}^{0} \ldots d_{k}^{0}}$. For simplicity we denote by $Q(x, n)$ the cardinality of above set.

By use of the ergodic theorem, the following result is established in [4.

Lemma 4. Let a $k$-tuple $\left(d_{1}^{0}, \ldots, d_{k}^{0}\right) \in\{0,1\}^{k}$ be fixed and let $Q(x, n)$ be defined as above. Then there exists a subset $F_{d_{1}^{0} \ldots d_{k}^{0}} \subseteq[0,1]$ satisfying the following conditions:

(i) $\mu\left(F_{d_{1}^{0} \ldots d_{k}^{0}}\right)=1$.

(ii) For any $x \in F_{d_{1}^{0} \ldots d_{k}^{0}}$, there are a positive integer $n_{0}$ and a constant $c$, both dependent of $x$, such that $Q(x, n) \geq c n, n \geq n_{0}$.

We are in a position to establish an equivalence for the pointwise convergence of a cascade sequence. 
Theorem 5. Suppose that the sequence $\left(\Phi_{n}\right)_{n=0}^{\infty}$ is bounded in $\left(L_{\infty}([0,1])\right)^{N}$. Then the following statements are equivalent:

(i) $\left(\Phi_{n}\right)_{n=0}^{\infty}$ converges a.e. on $[0,1]$.

(ii) For a.e. $x \in[0,1]$, there exist a positive constant $c$ and a constant $\gamma \in(0,1)$ such that $\left\|\Phi_{n+1}(x)-\Phi_{n}(x)\right\| \leq \gamma^{n}$ for sufficiently large $n$.

Proof. We only need to prove (i) $\Rightarrow$ (ii). Suppose (i) is true. By bounded dominated convergence theorem, the sequence $\left(\Phi_{n}\right)_{n=0}^{\infty}$ converges in $L_{1}([0,1])$. By a result of [1 there are a subset $E \subseteq[0,1]$ with $\mu(E)=1$ and a matrix norm satisfying $\left.|| A_{d}\right|_{V\left(S_{\left.E, \Phi_{0}\right)}\right.} \| \leq 1$ for $d=0,1$. For this matrix norm, Theorem 3 ensures the existence of some $k$ such that (9) holds for $B_{d}=\left.A_{d}\right|_{V\left(S_{F, \Phi_{1}-\Phi_{0}}\right)}$, where $F \subseteq[0,1]$ with $\mu(F)=1$. It implies that there exists a $k$ tuple $\left(d_{1}^{0}, \ldots, d_{k}^{0}\right)$ satisfying

$$
\eta:=\left\|B_{d_{1}^{0}} \cdots B_{d_{k}^{0}}\right\|<1 .
$$

By (6) we have for a.e. $x$

$$
\Phi_{n+1}(x)-\Phi_{n}(x)=A_{d_{1}(x)} \cdots A_{d_{n}(x)}\left(\Phi_{1}\left(\tau^{n} x\right)-\Phi_{0}\left(\tau^{n} x\right)\right) .
$$

Let $G=\bigcap_{n=0}^{\infty} \tau^{n}(F)$. Then $\mu(G)=1$ and $\tau^{n}(G) \subseteq G \subseteq F$ for any $n=1,2, \ldots$. Therefore, for any $\varepsilon_{1}, \ldots, \varepsilon_{m} \in\{0,1\}$ and $x \in G$, we have

$$
A_{\varepsilon_{1}} \cdots A_{\varepsilon_{m}}\left(\Phi_{1}\left(\tau^{n} x\right)-\Phi_{0}\left(\tau^{n} x\right)\right) \in V\left(S_{F, \Phi_{1}-\Phi_{0}}\right) .
$$

Suppose that $i$ satisfies $\tau^{i k} x \in T_{d_{1}^{0} \ldots d_{k}^{0}}$. Note that $\left.\left(A_{d_{1}^{0}} \cdots A_{d_{k}^{0}}\right)\right|_{V\left(S_{F, \Phi_{1}-\Phi_{0}}\right)}=$ $B_{d_{1}^{0}} \cdots B_{d_{k}^{0}}$. Then the block of product $A_{d_{i k+1}(x)} \cdots A_{d_{i k+k}(x)}$ has norm $\eta$ on $V\left(S_{F, \Phi_{1}-\Phi_{0}}\right)$.

We now consider the remaining $n-k Q(x, n)$ matrices in the product $A_{d_{1}(x)} \cdots$ $A_{d_{n}(x)}$. Lemma 2 tells us that each $A_{d_{i}(x)}$ has a norm not larger than 1 on $V\left(S_{E, \Phi_{0}}\right)$. On the other hand, for any $x \in E$, by construction of $E, \tau^{n} \in E \forall n=0,1, \ldots$. Therefore, for any $\varepsilon_{1}, \ldots, \varepsilon_{m} \in\{0,1\}$, we have

$$
\begin{aligned}
& A_{\varepsilon_{1}} \cdots A_{\varepsilon_{m}}\left(\Phi_{1}\left(\tau^{n} x\right)-\Phi_{0}\left(\tau^{n} x\right)\right) \\
= & A_{\varepsilon_{1}} \cdots A_{\varepsilon_{m}} A_{d_{n+1}(x)} \Phi_{0}\left(\tau^{n+1} x\right)-A_{\varepsilon_{1}} \cdots A_{\varepsilon_{m}} \Phi_{0}\left(\tau^{n} x\right) \in V\left(S_{E, \Phi_{0}}\right) .
\end{aligned}
$$

In summary, for any $x \in E \cap G$ we have

$$
\left\|\Phi_{n+1}(x)-\Phi_{n}(x)\right\| \leq \eta^{Q(x, n)}\left\|\Phi_{1}\left(\tau^{n} x\right)-\Phi_{0}\left(\tau^{n} x\right)\right\| .
$$

The statement (ii) follows from conclusion (ii) of Lemma 4. The proof is complete.

In the deduction of (i) $\Rightarrow$ (ii) from the proof of Theorem 5 , only the condition that $\left(\Phi_{n}\right)_{n=0}^{\infty}$ converges in $\left(L_{1}([0,1])\right)^{N}$ is needed. Therefore, we actually have proved the following result.

Corollary 6. Suppose that the sequence $\left(\Phi_{n}\right)_{n=0}^{\infty}$ is bounded in $\left(L_{\infty}([0,1])\right)^{N}$. Then $\left(\Phi_{n}\right)_{n=0}^{\infty}$ converges a.e. on $[0,1]$ if and only if for some $p \in[1, \infty),\left(\Phi_{n}\right)_{n=0}^{\infty}$ converges in $\left(L_{p}([0,1])\right)^{N}$. 


\section{Proof And generalization of Theorem 1}

Proof of Theorem 1. For a finitely supported refinement mask $a$, we assume without loss of generality that $a(\alpha)=0 \forall \alpha<1$ or $\alpha>N-1$, where $N$ is a positive integer. We construct two $N \times N$ matrices $A_{d}, d=0,1$, as follows:

$$
A_{d}=(a(2 \alpha-\beta+d))_{0 \leq \alpha, \beta \leq N-1} .
$$

For a compactly supported function $\phi_{0}$, we construct a cascade sequence $\left(\phi_{n}\right)_{n=0}^{\infty}$ by (2). It is easily seen that there exists an integer $n_{0}$ such that $\operatorname{supp} \phi_{n} \subseteq$ $[0, N] \forall n \geq n_{0}$.

Let $\Phi_{n}(x)=\left(\phi_{n}(x+\alpha)\right)_{\alpha=0}^{N-1}, x \in[0,1]$. Then they satisfy (55) with $A_{d}$ given above and for all $n \geq n_{0}+1$. Moreover, $\left(\Phi_{n}\right)_{n=0}^{\infty}$ is bounded in $L_{\infty}([0,1)]$. Clearly, Theorem 1 follows from Theorem 5 and Corollary 6 . The proof is complete.

We now discuss the pointwise convergence of $k$-th derivatives. Let $\left(\phi_{n}\right)_{n=0}^{\infty}$ be a cascade sequence constructed by (2). Then the sequence $\left(\phi_{n}^{(k)}\right)_{n=0}^{\infty}$ itself is also a cascade sequence with the mask $a=(a(\alpha))$ replaced by the mask $2^{k} a$. Theorem 1 applies in this case. We have established the following result.

Theorem 7. Let $\phi_{0}$ be a compactly supported function in $L_{\infty}(\mathbb{R})$ and let $k$ be a positive integer. Suppose that the sequence $\left(\phi_{n}^{(k)}\right)_{n=0}^{\infty}$ of $k$-th derivatives is bounded in $L_{\infty}(\mathbb{R})$. Then the following statements are equivalent:

(i) $\left(\phi_{n}^{(k)}\right)_{n=0}^{\infty}$ converges a.e. $x \in \mathbb{R}$.

(ii) For a.e. $x \in \mathbb{R}$, there exist a positive constant $c$ and a constant $\gamma \in(0,1)$ such that $\left|\phi_{n+1}^{(k)}(x)-\phi_{n}^{(k)}(x)\right| \leq c \gamma^{n} \forall n=1,2, \ldots$.

(iii) For some $p \in[1, \infty),\left(\phi_{n}^{(k)}\right)_{n=0}^{\infty}$ converges in $L_{p}(\mathbb{R})$.

We end the paper with an example.

Example 8. Let a mask $a$ be given by $\tilde{a}(z)=\frac{\left(1+z^{3}\right)^{k}}{2^{k-2}(1+z)}, k \geq 2$, where $\widetilde{a}$, for a finitely supported sequence $a$, is defined by $\widetilde{a}(z)=\sum_{\alpha \in \mathbb{Z}} a(\alpha) z^{\alpha}$. If we choose an initial function $\phi_{0}=\underbrace{\chi_{[0,3]} * \chi_{[0,3]} * \cdots * \chi_{[0,3]}}_{k \text { functions }}$, then the sequence $\left(\phi_{n}^{(k-2)}\right)_{n=1}^{\infty}$ is bounded in $L_{\infty}(\mathbb{R})$ and converges a.e. on $\mathbb{R}$. Consequently, there exists a refinable function $\phi$ corresponding to mask $a$ with $\phi^{(k-2)} \in L_{\infty}(\mathbb{R})$.

Proof. Let $b$ be a finitely supported sequence defined by $\widetilde{b}(z)=\left(1+z^{3}\right)^{k} / 2^{k-1}$. Then $\phi_{0}$ is a refinable function corresponding to $b$.

For any function $g$, it follows from $a(\alpha)+a(\alpha-1)=2 b(\alpha) \forall \alpha \in \mathbb{Z}$ that

$$
C_{a} g-C_{a} g(\cdot-1)=2\left(C_{b} g-C_{b} g(\cdot-1 / 2)\right) .
$$

By induction we have

$$
C_{a}^{n} g-C_{a}^{n} g(\cdot-1)=2^{n}\left(C_{b}^{n} g-C_{b}^{n} g\left(\cdot-2^{-n}\right)\right), \quad n=1,2, \ldots
$$

In particular we choose $g=\phi_{0}=\underbrace{\chi_{[0,3]} * \chi_{[0,3]} * \cdots * \chi_{[0,3]}}_{k \text { functions }}$. Then $\phi_{0}=C_{b}^{n} \phi_{0}$ and therefore $\phi_{n}=C_{a}^{n} \phi_{0}$ satisfies

$$
\phi_{n}-\phi_{n}(\cdot-1)=2^{n}\left(\phi_{0}-\phi_{0}\left(\cdot-2^{-n}\right)\right), \quad n=1,2, \ldots
$$


Since $\phi_{0}^{(k-1)} \in L_{\infty}(\mathbb{R})$, it follows from the last equality that

$$
\left\|\phi_{n}^{(k-2)}-\phi_{n}^{(k-2)}(\cdot-1)\right\|_{L_{\infty}(\mathbb{R})} \leq\left\|\phi_{0}^{(k-1)}\right\|_{L_{\infty}(\mathbb{R})} \quad \forall n=1,2, \ldots
$$

Moreover, for any $1 \leq p<\infty$, we have the following convergence in $L_{p}(\mathbb{R})$ :

$$
\phi_{n}^{(k-2)}-\phi_{n}^{(k-2)}(\cdot-1)=2^{n}\left(\phi_{0}^{(k-2)}-\phi_{0}^{(k-2)}\left(\cdot-2^{-n}\right)\right) \rightarrow \phi_{0}^{(k-1)} .
$$

Let $E \subset \mathbb{R}$ be a compact set. For any $f$ defined on $\mathbb{R}$ with $\operatorname{supp} f \subseteq E$, we define a function $g=f-f(\cdot-1)$. Then the functions $f(x+\alpha), \alpha \in \mathbb{Z}$, may be represented in terms of $g(\cdot-\beta), \beta \in \mathbb{Z}$, as $f(x+\alpha)=\sum_{\beta \geq 0} g(x+\alpha-\beta)$. The series converges due to the fact that $g$ is a compactly supported function. By the equality

$$
\|f\|_{L_{p}(\mathbb{R})}^{p}=\sum_{\alpha \in \mathbb{Z}} \int_{0}^{1}|f(x+\alpha)|^{p} d x=\sum_{\alpha \in \mathbb{Z}} \int_{0}^{1}\left|\sum_{\beta \geq 0} g(x+\alpha-\beta)\right|^{p},
$$

we can find a constant $c$, dependent of $E$ and $p$, such that for any $f \in L_{p}(\mathbb{R}), 1 \leq$ $p<\infty$, with $\operatorname{supp} f \subseteq E$

$$
\|f\|_{L_{p}(\mathbb{R})} \leq c\|g\|_{L_{p}(\mathbb{R})} .
$$

As is known, $\left(\phi_{n}^{(k-2)}-\phi_{n}^{(k-2)}(\cdot-1)\right)_{n=0}^{\infty}$ is a Cauchy sequence in $L_{p}(\mathbb{R}), 1 \leq p<$ $\infty$, so is $\left(\phi_{n}^{(k-2)}\right)_{n=0}^{\infty}$ by (14).

Clearly, when $p=\infty$, there exists a constant $c$ such that the inequality (14) holds for any $f$ with $\operatorname{supp} f \subseteq E$. Therefore, for $n=1,2, \ldots,\left\|\phi_{n}^{(k-2)}\right\|_{L_{\infty}(\mathbb{R})} \leq$ $c\left\|\phi_{0}^{(k-1)}\right\|_{L_{\infty}(\mathbb{R})}$ by (13) and (14). The conclusion of the example follows from Theorem 7 .

It was proved in [11] by a different method that there exists a refinable function in $L_{p}(\mathbb{R}), 1 \leq p<\infty$, corresponding to mask $a$ when $k=2$.

\section{REFERENCES}

[1] M. A. Berger and Y. Wang, Bounded semi-groups of matrices, Lin. Alg. Appl., 166(1992), 21-27. MR1152485 (92m:15012)

[2] A. S. Cavaretta, W. Dahmen, and C. A. Micchelli, Stationary Subdivision, Memoirs of AMS, V. 453, 1991. MR1079033 (92h:65017)

[3] D. R. Chen, Construction of smooth refinable vectors by cascade algorithms, SIAM Numer. Anal., 40(2002), 1354-1368. MR1951898(2004b:42074)

[4] D. R. Chen, Local regularity of $L_{\infty}$-refinable function vectors, J. Fourier Anal. Appl., 11 (2005), 654-667. MR2190677

[5] D. R. Chen and M. Han, Convergece of cascade sequence for arbitray refinement mask and individual initial function, Sciences in China (Ser. A), 35(2005), 78-86.

[6] D. R. Chen, R. Q. Jia, and S. D. Riemenschneider, Vector subdivision schemes in Sobolev spaces, Appl. Comput. Harmonic Anal., 12 (2002), 128-149. MR.1874918(2002k:65220)

[7] D. R. Chen and G. Plonka, Convergence of cascade algorithms in Sobolev spaces for perturbed refinement masks, J. Approx. Theory, 119(2002), 133-155. MR1939279 (2003h:42049)

[8] B. Han, and R. Q. Jia, Multivariate refinement equations and convergence of subdivision schemes. SIAM J. Math. Anal. Appl., 29 (1998), 1177-1199. MR.1618691 (99f:41018)

[9] R. Q. Jia, Subdivision schemes in $L_{p}$ spaces, Adv. in Comput. Math., 3 (1995), 309-341. MR1339166 (96d:65028)

[10] R. Q. Jia, Characterization of smoothness of multivariate refinable functions in Sobolev spaces, Trans. Amer. Math. Soc., 351 (1999), 4089-4112. MR.1473444 (99m:42050)

[11] R. Q. Jia, K. S. Lau, and D. X. Zhou, $L_{p}$ solutions of refinement equations, J. Fourier Anal. Appl., 7 (2001), 143-167. MR1817673(2002i:42049)

[12] R. Q. Jia, S. D. Riemenschneider, and D. X. Zhou, Vector subdivision schemes and multiple wavelets, Math. Comp., 67 (1998), 1533-1563. MR.1484900 (99d:42062) 
[13] R. Q. Jia, S. D. Riemenschneider, and D. X. Zhou, Smoothness of multiple refinable functions and multiple wavelets. SIAM J. Matrix Anal. Appl., 21 (1999), 1-28. MR.1709723 (2000k:42050)

[14] W. Lawton, S. L. Lee, and Z. Shen, Convergence of multidimensional cascade algorithm, Numer. Math., 78(1998), 427-438. MR1603354 (98k:41027)

[15] S. Li, Convergence of cascade algorithms in Sobolev spaces associated with multivariate refinement equations, J. Math. Anal. Appl., 257(2001), 154-169. MR1824672(2002c:42053)

[16] R. L. Long and Q. Mo, $L^{2}$-convergence of vector cascade algorithm, Approx. Theory Appl. (N.S.), 15(1999), No.4, 29-49. MR.1747327 (2001c:65178)

[17] C. A. Micchelli, Mathematical Aspects of Geometric Modeling, CBMS 65, SIAM, Philadelphia, 1995. MR 1308048 (95i:65036)

[18] C. A. Micchelli and T. Sauer, On vector subdivision, Math. Z., 229(1998), 621-674. MR.1664782 (2000d:42016)

[19] G.-C. Rota and G. Strang, A note on the joint spectral radius, Indag. Math., 22 (1960), 379-381. MR0147922(26:5434)

[20] Z. Shen, Refinable function vectors, SIAM Math. Anal., 29 (1998), 235-250. MR.1617183 (99d:41038)

[21] G. Strang, Eigenvalues of $(\downarrow) H$ and convergence of the cascade algorithm, IEEE Signal Process., 44(1996), 233-238.

[22] Y. Wang, Two-scale dilation equations and the mean spectral radius, Random and Computational Dynamics, 4 (1996), 49-72. MR1376114 (96j:42023)

Department of Mathematics, and LmiB, Beijing University of Aeronautics and AeStronautics, Beijing 100083, People's Republic of China

Department of Mathematics, and LmiB, Beijing University of Aeronautics and AeStronautics, Beijing 100083, People's Republic of China 